\title{
Rheological Properties in Aqueous Solution for Hydrophobically Modified Polyacrylamides Prepared in Inverse Emulsion Polymerization
}

\author{
Shirley Carro, ${ }^{1}$ Valeria J. Gonzalez-Coronel, ${ }^{2}$ Jorge Castillo-Tejas, ${ }^{1}$ \\ Hortensia Maldonado-Textle, ${ }^{3}$ and Nancy Tepale ${ }^{2}$ \\ ${ }^{1}$ Facultad de Ciencias Básicas, Ingeniería y Tecnología, Universidad Autónoma de Tlaxcala, Calzada Apizaquito S/N, \\ 90300 Apizaco, TLAX, Mexico \\ ${ }^{2}$ Facultad de Ingeniería Química, Benemérita Universidad Autónoma de Puebla, Av. San Claudio y 18 Sur, \\ Col. San Manuel Ciudad Universitaria, 72520 Puebla, PUE, Mexico \\ ${ }^{3}$ Centro de Investigación en Química Aplicada, Blvd. Enrique Reyna \#140, 25253 Saltillo, COAH, Mexico
}

Correspondence should be addressed to Valeria J. Gonzalez-Coronel; valeria.gonzalez@correo.buap.mx

Received 6 October 2016; Revised 2 February 2017; Accepted 6 February 2017; Published 29 March 2017

Academic Editor: Atsushi Sudo

Copyright (C) 2017 Shirley Carro et al. This is an open access article distributed under the Creative Commons Attribution License, which permits unrestricted use, distribution, and reproduction in any medium, provided the original work is properly cited.

\begin{abstract}
Inverse emulsion polymerization technique was employed to synthesize hydrophobically modified polyacrylamide polymers with hydrophobe contents near to feed composition. Three different structures were obtained: multisticker, telechelic, and combined. NDimethyl-acrylamide (DMAM), n-dodecylacrylamide (DAM), and n-hexadecylacrylamide (HDAM) were used as hydrophobic comonomers. The effect of the hydrophobe length of comonomer, the initial monomer, and surfactant concentrations on shear viscosity was studied. Results show that the molecular weight of copolymer increases with initial monomer concentration and by increasing emulsifier concentration it remained almost constant. Shear viscosity measurements results show that the length of the hydrophobic comonomer augments the hydrophobic interactions causing an increase in viscosity and that the polymer thickening ability is higher for combined polymers.
\end{abstract}

\section{Introduction}

Water-soluble associating polymers have been studied extensively due to their rheological characteristics in aqueous solution. These materials are structured by a water-soluble backbone where a small amount of hydrophobic groups is inserted, distributed randomly or as microblocks, in the main polymer chain (multisticker), at both extremes of it (telechelic) or along the macromolecular chain as well as the chain ends (combined) $[1,2]$. Associating polymers can be synthesized by modifying, chemically, a precursor polymer or by free radical copolymerization of the suitable monomers [3]. For the latter method, copolymers based on acrylamide (AM) and its derivatives have been widely researched. Different synthesis techniques have been explored: (i) solution polymerization, where the hydrophobic and hydrophilic monomers are solubilized by the addition of a cosolvent; the resultant polymer can be maintained as soluble (homogeneous polymerization) $[4,5]$ or insoluble (precipitation polymerization) [5-7] in the reaction medium; (ii) heterogeneous polymerization, where the water-insoluble monomer is distributed finely in the acrylamide aqueous medium under stirring $[4,8,9]$; (iii) micellar polymerization, where, in presence of a surfactant, due to the amphiphilic character of its molecules, the hydrophobic monomer is solubilized within the micelles formed [3, 10-12]. This last method is the most employed because the copolymers prepared have a hydrophobic blocky distribution along the hydrophilic chain and it is possible to control the length of such microblocks. Besides, the copolymers obtained have an excellent thickening ability.

For many applications, molecular weight of associative polymers is required to be sufficiently high. Solution 
polymerization processes are inadequate because, as conversion increases, viscosity in reaction medium extremely augments, causing mixing and heat transfer problems [13]. Inverse emulsion and microemulsion polymerization have been also employed to synthetize acrylamide copolymers [11, 14-16]. Inverse emulsion polymerization is one of the best methods for obtaining polymers with high molecular weight and low viscosity of the medium of reaction. In the acrylamide polymerization, the main locus of particle formation is the monomer droplets [17-20]; however, if an oilsoluble initiator is employed, a certain amount of micellar or homogeneous nucleation can be present [19]. Polymerization occurs near the disperse phase interface, monomer droplets, because of the formation of a contrasting boundary between oil and aqueous phases. Consequently, the copolymerization of monomers with strongly dissimilar hydrophilicities is more probable [21]. Thus, polymerization in inverse emulsion is an attractive alternative to micellar polymerization to synthesize hydrophobically modified polyacrylamide.

In this work, hydrophobically modified polyacrylamides were synthesized in inverse emulsion polymerization varying the surfactant and monomer concentration. The microstructure obtained and the reaction mechanism are proposed, taking as base the rheometric behavior of these materials. We describe a detailed study of the rheological properties in aqueous solutions of three different structures of hydrophobically modified polyacrylamides (telechelic, multisticker, and combined) prepared using two different hydrophobic linear groups, of 12 and 16 carbon atoms.

\section{Experimental Section}

2.1. Materials. All substances were employed without additional purification. Acrylamide (AM, Aldrich 99\% purity) was used as hydrophilic monomer and n-dimethyl acrylamide (DMAM, Aldrich 99\% purity), n-dodecyl acrylamide (DAM), and n-hexadecyl acrylamide (HDAM) as hydrophobic comonomers. 4,4'-Azobis(4-cyanovaleric acid) (ACVA, Aldrich 99\% purity) was used as initiator and SPAN $80^{\circledR}$ (nonionic emulsifier, sorbitan monooleate, Aldrich) as surfactant. DAM and HDAM were prepared according to [22]. For telechelic and combined polymers, hydrophobically modified initiator, $\mathrm{ACVA}_{12}$ or $\mathrm{ACVA}_{16}$, was used; the synthesis of these initiators was previously reported [2]. Toluene (Aldrich) and acetonitrile (Aldrich) were employed as organic phase in emulsion and solution polymerization, respectively. In Figure 1, a schematic representation of the synthesized copolymers is presented.

2.2. Polymerization. Batch copolymerization processes were carried out at $80^{\circ} \mathrm{C}$, using a constant temperature water bath, in a $500 \mathrm{ml}$ glass reactor equipped with mechanical stirrer. For solution polymerization, monomers, $3 \mathrm{wt}$ \% (99\% mol $\mathrm{AM}$ and $1 \%$ mol hydrophobic comonomer), were dissolved in acetonitrile. For emulsion polymerization, the continuous phase (51 wt.\%) was prepared dissolving the hydrophobic comonomer and surfactant (4.5 or $36 \mathrm{wt} \%$ relative to monomer feed) in toluene. Dispersed phase, 49 wt.\%, was prepared dissolving AM in deionized and distilled water and added to organic phase. Total monomer concentration was varied, $\left[M_{0}\right], 1.5$ or $4 \mathrm{wt} . \%$. Molar monomer relation was maintained as in solution polymerization. Stirrer was adjusted at $600 \mathrm{rpm}$. After homogenization, once reaching temperature reaction, the mixture was purged with nitrogen, at least 20 minutes before adding initiator $0.002 \mathrm{M}$ that had been previously dissolved in the continuous phase, toluene or acetonitrile, and heated to the temperature reaction. The reactions were carried out for $1 \mathrm{~h}$. The nitrogen atmosphere was maintained during the polymerization.

2.3. Characterization. Samples were withdrawn periodically from the reactor for the conversion and molecular weight analysis. The samples were short-stopped with a hydroquinone solution (0.4 wt.\%). As polymerization progresses, the polymer obtained appeared as a viscous solution or a gel (for the highest monomer contents). In order to remove unreacted monomers and surfactant, samples and final polymer were precipitated and washed into a methanol/acetone mixture (50:50 vol./vol.). Finally, samples were dried to constant weight in oven at $50^{\circ} \mathrm{C}$. Conversion was followed by gravimetric technique.

2.3.1. Hydrophobic Monomer Composition. The hydrophobic monomer composition in the copolymers was determined by $1 \mathrm{H}-\mathrm{NMR}$ spectroscopy at room temperature in a JEOL $300 \mathrm{MHz}$ spectrometer; $\mathrm{D}_{2} \mathrm{O}-\mathrm{DMSO}(85-15 \% \mathrm{wt})$ was used for associative polymers. This kind of characterization has been performed for associative polymers elsewhere [23-26].

2.3.2. Molecular Weight. The hydrophobic copolymers cannot be characterized by size exclusion chromatography (SEC) in water, due to the association of the hydrophobic groups, as it has occurred elsewhere [1]. However, molecular weight of the homopolymer from AM, prepared under identical experimental conditions, was measured in a Hewlett Packard HPLC series 1100 equipped. Calibration was carried out with standards of polyethylene glycol and polyethylene oxide and a buffer solution at $\mathrm{pH}=7$ as eluent to a flow rate of $1 \mathrm{~mL} / \mathrm{min} . M_{n}$ was 520000 with a polydispersity index $M_{w} / M_{n}$ of 2.0. Viscometric molecular weight was determined and reported as a valid approximation as it has been done elsewhere [27-30]. The viscosity-average molecular weight, $M_{v}$, of copolymers was calculated from

$$
[\eta]=6.31 \times 10^{-3} M_{v}^{0.8}\left(\mathrm{~mL} \cdot \mathrm{g}^{-1}\right) .
$$

Parameters in (1) are proper for AM [31]; nevertheless, they were used to estimate the copolymers $M_{v}$ value. Limiting viscosity number $[\eta]$ was determined with an Ubbelohde viscometer in distilled deionized water at $25^{\circ} \mathrm{C}$.

2.3.3. Rheological Measurements. Samples preparation was performed as follows: a specific amount of polymer was dissolved in deionized water. These solutions were put into agitation during 48 hours. More concentrated solutions containing polymers with a larger hydrophobic chain were 
<smiles>CC(C#N)(CCC(=O)O)CC(CC(C)(C#N)CCC(=O)O)C(N)=O</smiles>

(a)

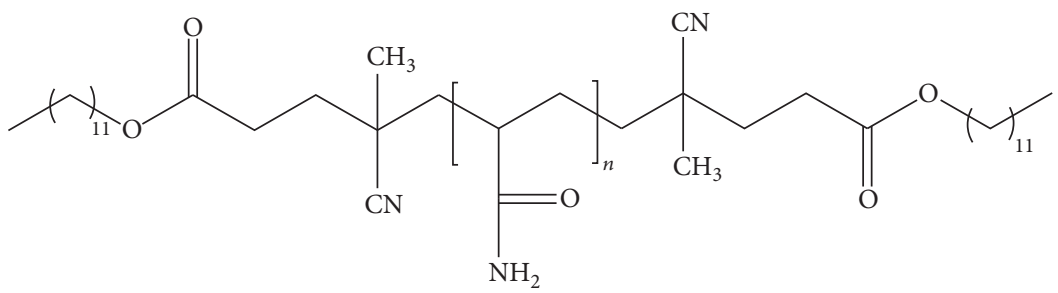

(b)<smiles>CC(CC(C)C(C)C(C)CC(C)(C#N)CCC(=O)O)CC(C)(C#N)CCC(=O)O</smiles>

(c)

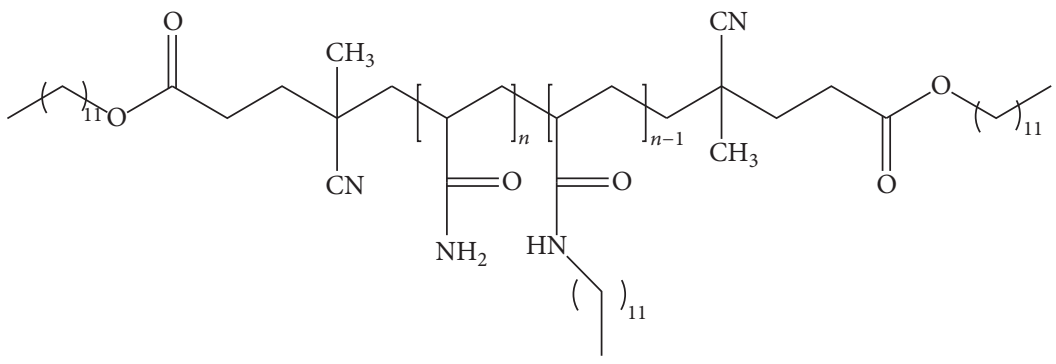

(d)

FIGURE 1: Schematic representation of the synthesized hydrophobic polymers: (a) homopolymer, (b) telechelic, (c) multisticker, and (d) combined.

stirred until they were homogeneous. Interval concentration studied was 0.1 to $10 \%$ wt.

Solutions were measured in two rheometers, depending on the concentration of the sample. The more diluted samples were analyzed in a rotational rheometer Rheolab QC, using geometry double-walled cylinder (DG42) and more concentrated samples at the Physica UDS200 controlled stress rheometer equipped with a cone and plate (angle 2 and diameter $50 \mathrm{~mm})$ at $25 \pm 0.05^{\circ} \mathrm{C}$. The zero-shear viscosity $\left(\eta_{o}\right)$ was obtained by extrapolation of the apparent viscosity.

Sample Code. The name assigned to the polymers is according to hydrophobic monomer and initiator. For example,
PAM-co-DAM/12 relates to the copolymer poly(acrylamideco-dodecilacrylamide) synthesized by copolymerizing acrylamide and the hydrophobic monomer dodecylacrylamide using hydrophobically modified initiator $\mathrm{ACVA}_{12}$. This copolymer corresponds to a combined type.

\section{Results and Discussion}

In order to demonstrate that inverse emulsion polymerization is a suitable technique to obtain associative polymers, results for multisticker copolymers are compared with solution polymerization. Viscosity results intend to show the advantage of obtaining higher molecular weight with emulsion 
TABLE 1: Viscometric molecular weight for the different polymers synthetized.

\begin{tabular}{|c|c|c|c|c|}
\hline Method & Solution & & & Emulsion \\
\hline Monomer concentration* wt $\%$ & 3 & & & 4 \\
\hline \multirow{2}{*}{ Polymer } & \multicolumn{4}{|c|}{ Molecular weight (g/mol) } \\
\hline & Viscometric & Viscometric & SEC & Viscometric \\
\hline PAM & 55933 & 216520 & $\begin{array}{l}M_{n}=137,500 \\
M_{w}=506490\end{array}$ & 466029 \\
\hline PAM-co-DAM & 80879 & 239777 & - & 492249 \\
\hline PAM-co-DMA & - & - & - & 514452 \\
\hline PAM-co-HDAM & - & - & - & 350763 \\
\hline
\end{tabular}

${ }^{*}$ Monomer concentration is related to total weight of reaction mixture.

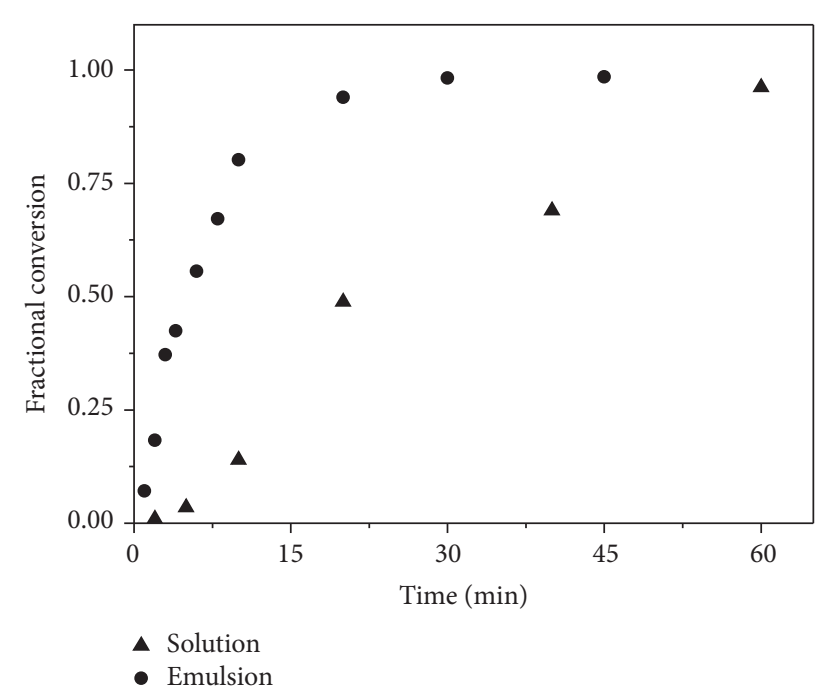

FIGURE 2: Evolution of fractional conversion for the PAM synthesized by emulsion and solution polymerization.

polymerization. Also, the effect of the hydrophobic monomer length and surfactant concentration is presented. The study for the rheological properties includes telechelic, multisticker, and combined copolymers.

3.1. Effect of the Hydrophobic Monomer Length. A comparison for the evolution of conversion between solution and emulsion AM homopolymerization is presented in Figure 2. As it can be seen, maximum conversion for emulsion polymerization is reached at really low time $(20 \mathrm{~min})$ in comparison with solution polymerization. Also, molecular weight is higher (Table 1), even though a smaller percentage of monomer is used in comparison with solution (1.5 versus 3 wt.\%). This behavior is expected, because of compartmentalization of the reaction loci [32]. Results show that the conditions employed provide an emulsion system. $M_{v}$ obtained by the homopolymers and copolymers are presented in Table 1. As it can be seen, $M_{v}$ value for PAM is between $M_{n}$ and $M_{w}$ obtained by SEC; then, results for $M_{v}$ or copolymers can be used as an approximation.

An interesting observation is that an increase in monomer concentration causes an increase in $M_{v}$ for emulsion polymerization. This behavior has been observed in styrene emulsion polymerization and was attributed to limited particle coagulation causing an apparent change in radical concentration, leading to bimolecular termination [33, 34]. In our case, for an increase in initial monomer concentration, $\left[M_{0}\right]$, a greater number in droplets is expected; however, for the same surfactant concentration, droplet degradation can take place via coalescence, or particle coagulation occurs because of a lack of surfactant to stabilize the system. As a consequence, a minor number of bigger droplets are obtained. Taking into consideration that in inverse emulsion polymerization monomer droplets are the sites of particle formation [17], more radicals can coexist leading to bimolecular termination. In this sense, the number and size of reaction loci can be manipulated, taking into consideration the stability system, to influence molecular weight. Higher monomer concentrations were tried; however, the viscosity of the medium reaction was too high that a gel was rapidly formed.

The difference in the polymer molecular weight is reflected in rheological behavior (Figure 3(a)). An increase in molecular mass results in a larger coil and more chain entanglements, which can be seen as increase in viscosity. Notice that when AM homopolymer is prepared by emulsion polymerization, its viscosity is three orders of magnitude greater than the polymer obtained by solution, even when the total monomer concentration is lower than that employed in solution polymerization. Thus, viscosity of these kinds of materials synthesized by inverse emulsion polymerization can be manipulated by adjusting the initial polymerization loci. Notice that a diminution in viscosity is observed as shear rate is increased for AM homopolymer synthesized by emulsion technique at higher monomer concentration. This behavior is typical for polymers with high molecular weight. Also, the behavior of multisticker copolymers synthesized by different methods is presented (Figure 3(b)). As it can be seen, the copolymer has a higher viscosity for all polymerization processes, and, again, molecular weight plays an important role; the larger the value for $M_{v}$, the higher the solution viscosity.

The $1 \mathrm{H}$ NMR spectra of the (a) homopolymer, (b) multisticker polymer PAM-co-DAM, (c) telechelic polymer $\mathrm{PAM} / 12$, and the (d) combined polymers are presented in Figure 4 . The chemical shifts are presented in part per million 
TABLE 2: Percentages of hydrophobic monomer incorporated for emulsion polymers, $4 \%$ solids contents.

\begin{tabular}{|c|c|c|c|c|}
\hline Type & Polymer & {$[H](\% \mathrm{~mol})$} & Conversion\% & $M_{v}(\mathrm{~g} / \mathrm{mol})$ \\
\hline Homopolymer & PAM & & 96 & 466029 \\
\hline \multirow{2}{*}{ Multisticker } & PAM-co-DAM & 0.80 & 94 & 492249 \\
\hline & PAM-co-HDAM & 0.85 & 97 & 350763 \\
\hline \multirow{2}{*}{ Telechelic } & $\mathrm{PAM} / 12$ & & 96 & 522211 \\
\hline & $\mathrm{PAM} / 16$ & & 89 & - \\
\hline \multirow{2}{*}{ Combined } & PAM-co-DAM/12 & 0.85 & 90 & 531107 \\
\hline & PAM-co-HDAM/16 & 0.82 & 92 & - \\
\hline
\end{tabular}

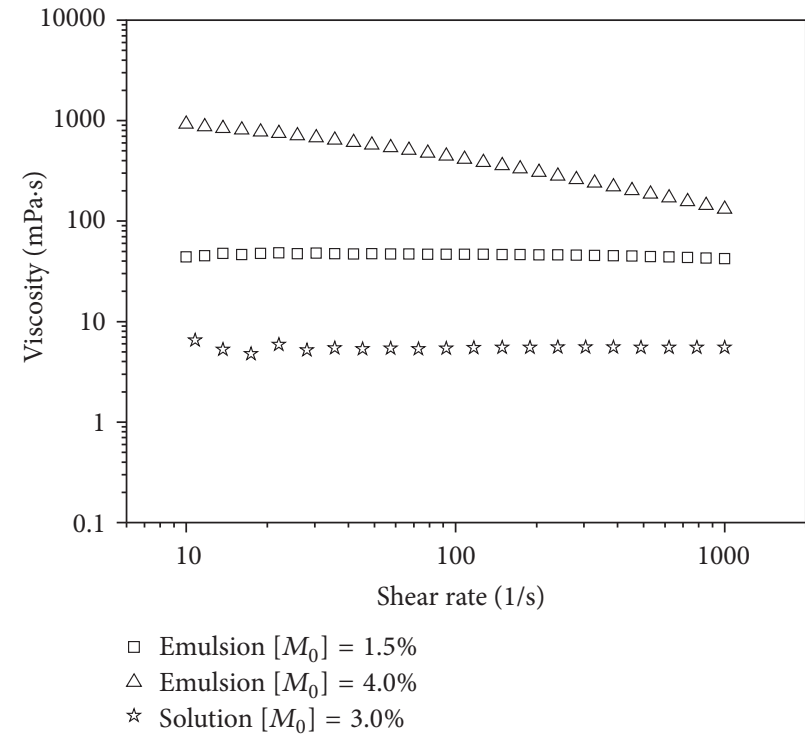

(a)

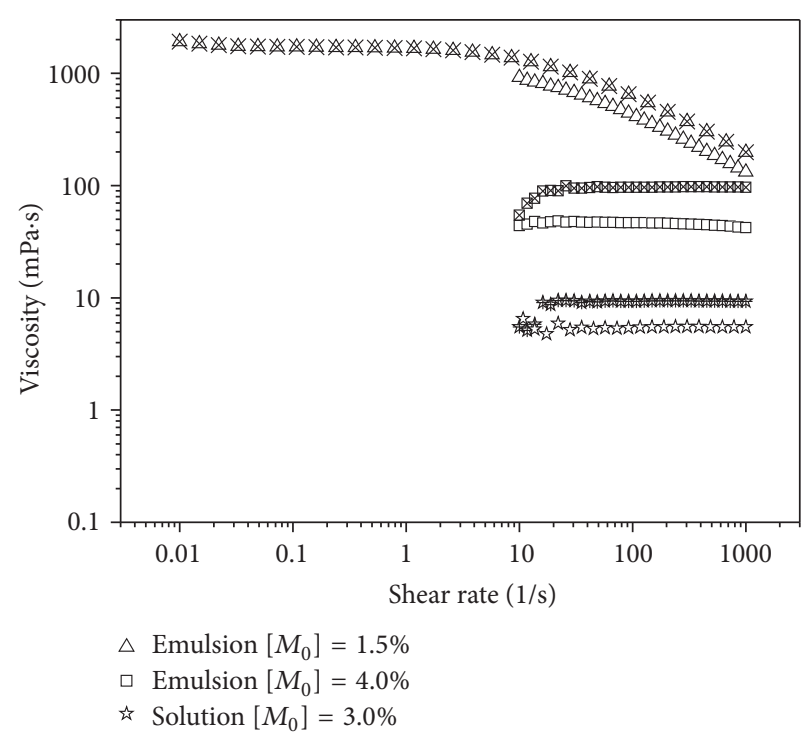

(b)

FIGURE 3: Influence of the polymerization method in the viscosity as a function the shear rate for (a) PAM and (b) HDAM-AM. Polymer concentration $5 \mathrm{wt} \%$ for aqueous solutions. Noncrossed symbols correspond to AM homopolymer. $\left[M_{0}\right]=$ initial monomer concentration.

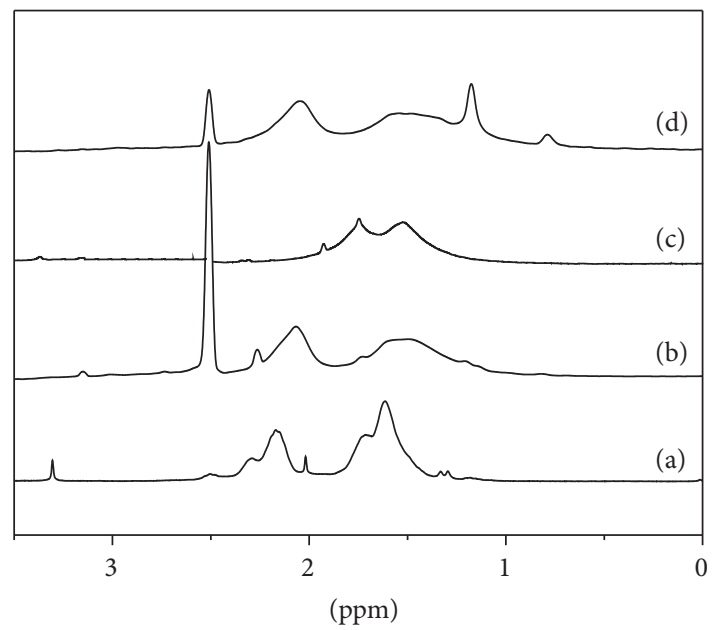

FIGURE 4: 1H NMR spectra of the polymers: (a) homopolymer PAM in $\mathrm{D}_{2} \mathrm{O}$, (b) multisticker PAM-co-DAM, (c) telechelic PAM/12, and (d) combined PAM-co-DAM/12 in $\mathrm{D}_{2} \mathrm{O}$-DMSO (85-15\%). downfield from the internal TMS standard or with respect to a solvent resonance line. The ratio of the two monomers in the copolymer was determined by integrating the signals of the methyl proton $\left({ }^{*} 0.8 \mathrm{ppm}\right)$ and of the ethylene proton attached to the backbone (1.1-1.8 ppm); the ratio was found to be around $0.80 \mathrm{~mol} \%$ with respect to the feed composition for multisticker and $0.85 \mathrm{~mol} \%$ for combined polymers. As it can be observed in the spectra for telechelic polymer, the corresponding signal to the hydrocarbon chain is not visible because of its low concentration in the polymer chain. Due to the low concentration of hydrophobic monomer, the signal in the spectrum is very tenuous. However, an approximation on that area can be carried out and properly be integrated [2326].

Table 2 shows the percentages of hydrophobic monomer incorporated for different copolymer structures. It also shows the obtained values for $M_{v}$. Notice that they are greater than those observed in similar polyacrylamides synthesized by solution polymerization, and the reported molecular 


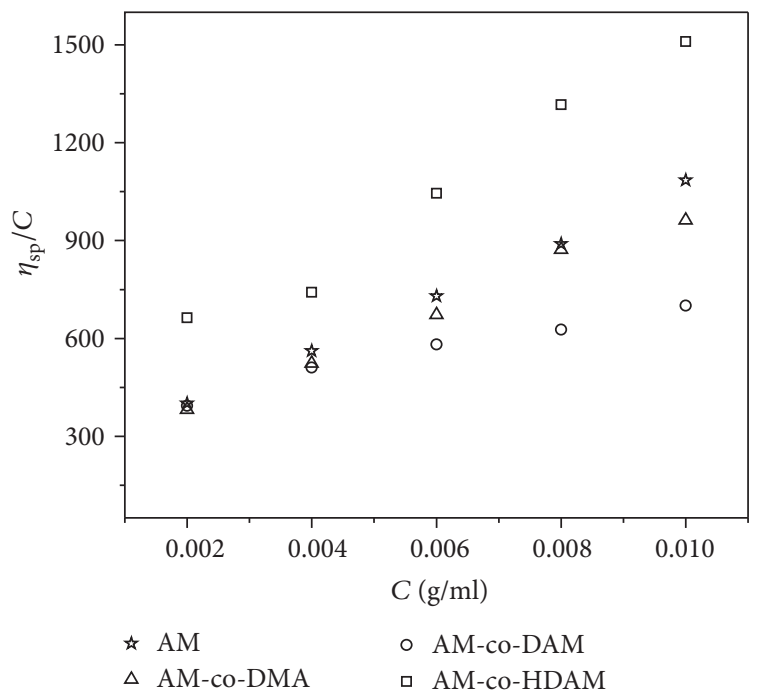

(a)

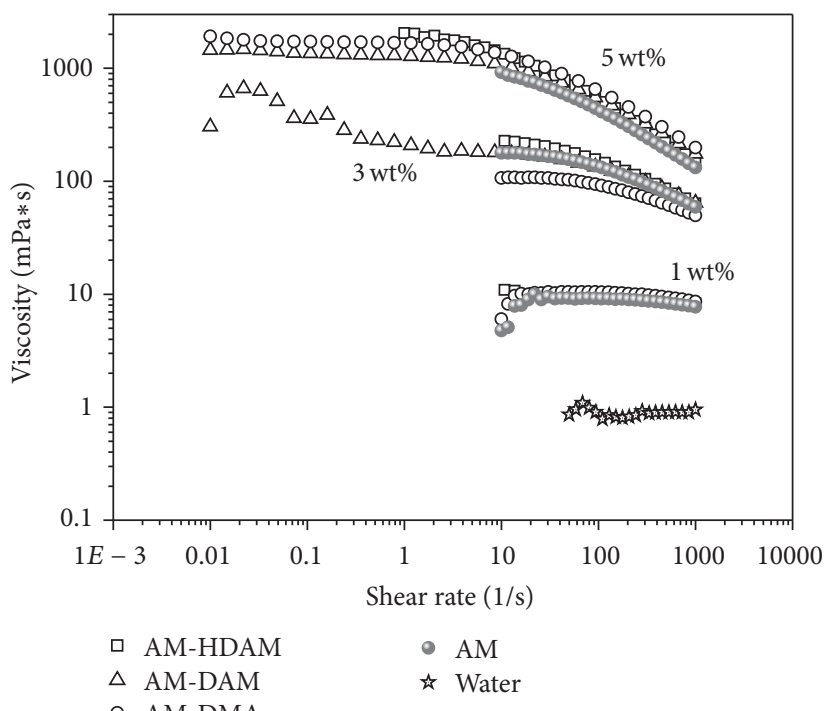

(b)

Figure 5: Influence of hydrophobic monomer length in (a) specific viscosity and (b) shear viscosity. Polymer concentration is indicated in the figure. Water viscosity is shown as reference.

weight for them was $200,000 \mathrm{~g} / \mathrm{mol}$, approximately [2, 35]. Results show that hydrophobe contents are near to the feed composition. These results demonstrate that inverse emulsion polymerization is a suitable technique to synthetize hydrophobically modified polyacrylamide polymers.

The behavior for specific viscosity, $\eta_{\mathrm{sp}}$, for the multisticker copolymers prepared by emulsion polymerization is shown in Figure 5(a). Results correspond to $\left[M_{0}\right]=4.0 \mathrm{wt} . \%$. The intention of using $\eta_{\mathrm{sp}}$ instead of shear viscosity is to compare the behaviors of the copolymers in a very dilute aqueous solution. Notice that as the length of hydrophobic alkyl chains increases, an augment in solutions viscosity is observed, even when the concentration of hydrophobic comonomer is low ( $1 \% \mathrm{~mol}$ ). Because of this low comonomer concentration, molecular weight does not change significantly compared with homopolymer (Table 1). However, the augment observed in $\eta_{\text {sp }}$ for the copolymer PAM-co-HDAM suggests hydrophobic associations. For the copolymer PAM-co-DAM, $\eta_{\mathrm{sp}}$ is slightly depressed because of the well-known phenomena of contraction at this dilute regimen of concentration due to intramolecular associations [36, 37]. At low solution concentrations, the possibility of interaction between different hydrophobic groups in polymer molecules is small. Intraaggregation associations predominate which results in a reduced coil size [36]. Therefore, at such concentrations, the specific viscosity of copolymers is often lower than their unmodified homopolymers.

Figure 5(b) shows the effect of polymer concentration in the steady-state viscosity, $\eta$, as a function of shear rate, and $\dot{\gamma}$, for the multisticker copolymers at different aqueous solutions concentration. For the lowest polymer solution concentration tested (1\%), the behavior is Newtonian; this is, there is no variation in viscosity with $\dot{\gamma}$, but as concentration augments $\eta$ increases drastically and the solutions exhibit a shearthinning behavior.

As it can be observed, variation between $\eta$ values corresponding to copolymers and homopolymer is not large. This behavior has previously been observed for comonomers with low hydrophobic length [38, 39]; however, HDAM has a longitude of 16 carbons. Besides, it was found that copolymers PAM-co-HDAM were water insoluble, which was not our case. A possible explanation in behavior for the polymers synthetized here and in other works is the difference in reaction sites. In the micellar copolymerization process, the hydrophobic monomer is solubilized within surfactant micelles whereas the hydrophilic monomer is dissolved in the aqueous continuous phase. This situation contrasts with the inverse emulsion process, where the hydrophobe is present in the continuous phase and the hydrophile in the dispersed phase. In the first case, blocks of hydrophobic comonomer are inserted along the hydrophilic chain. As surfactant concentration increases, the hydrophobic blocks length decrease and, consequently, viscosity diminishes because of the "dilution" of hydrophobic monomer in a mayor number of micelles. In inverse emulsion, the initiating radicals are formed by the decomposition of the hydrophobic initiator. These radicals cannot enter the hydrophilic polymer particles; however, they can add monomer units from the AM dissolved in the continuous phase. When radicals become more hydrophilic, they can enter the droplets and polymerize [40]. Besides, it has been suggested that AM is present in the droplets surface, behaving as cosurfactant [41, 42]; then oligoradicals can reach this monomer at the surface and be adsorbed. Also, as in micellar process, at the same time, hydrophobic comonomer and AM dissolved in the continuous phase are polymerized in a solution process; 


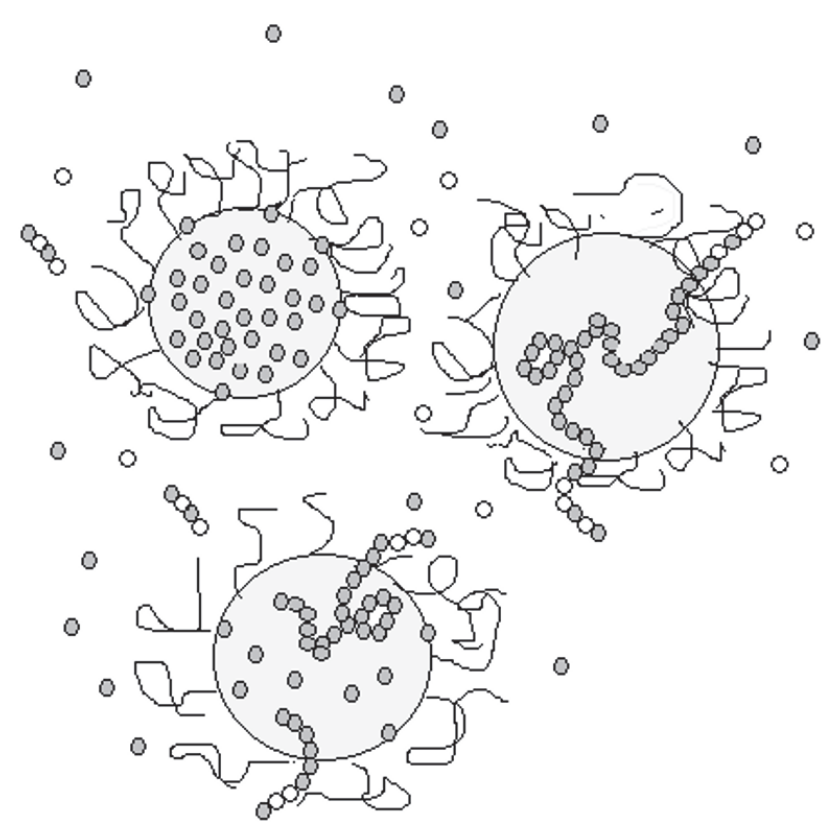

- Water soluble monomer

○ Hydrophobic monomer

FIGURE 6: Schematic representation of the inverse emulsion polymerization.

then, a random distributed copolymer is obtained [4]. In addition, due to the low concentration of the hydrophobic comonomer ( $1 \%$ mol solved in the continuous phase), it seems difficult to form blocks. Therefore, inverse emulsion process provides long AM chains, which are formed within monomer droplets, with both ends formed by a block of a hydrophobic-hydrophilic copolymer, randomly distributed, polymerized in the continuous phase (Figure 6).

If $\eta$ in Newtonian plateau is analyzed, the effect of the hydrophobic monomer length is clear; viscosity increases as hydrophobicity does: PAM $<$ DMA $<$ DAM $<$ HDAM. For solutions at $1 \mathrm{wt} . \%$, this effect is not appreciable but for solutions with $5 \mathrm{wt} . \%$ of polymer the effect is perceptible.

In micellar copolymerization, it has been proved that as surfactant concentration increases, the viscosity of the aqueous solutions for the hydrophobic copolymers decreases, due to a decrease in hydrophobic block length. The same monomer concentration is distributed in more micelles. However, for the copolymers obtained here, when a minor concentration of surfactant is employed, viscosity is higher. It could be attributed to the localization of the hydrophobic groups. As it was suggested, in the continuous phase, copolymerization like solution process is performed. Then, a statistical copolymer AM-HDAM is added to a large block of PAM (Figure 6). If reaction sites are decreased, then molecular weight inside the site of polymerization (monomer droplets) is increased; this block becomes larger and the hydrophobic groups are far from each other. In this case, the AM blocks can bend and the HB groups can associate easily. In this sense, the effect of initial surfactant concentration was investigated.

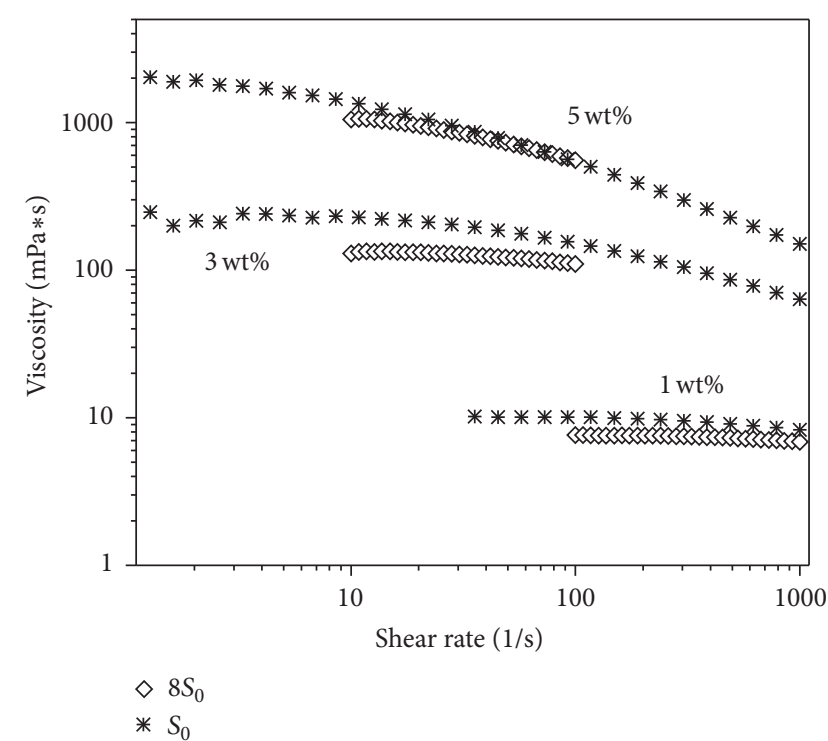

FIGURE 7: Influence of initial tensioactive concentration on shear viscosity.

Capek [32] found that the AIBN-initiated polymerization $R_{p}$ and molecular weight decrease at higher emulsifier concentrations. He argued that the decrease in the polymerization rate with increasing the emulsifier concentration is expected, because monomer concentration at the particle loci (diluted by the emulsifier molecules) diminishes. On the other hand, when a water-soluble initiator (ammonium persulfate) is used [43], the molecular weight of PAM is nearly independent of surfactant concentration. It is attributed to an increased number of polymer particles, which is followed by the decreased termination rate. Barari et al. [44] found that the molecular weight of final polymer increases by increasing the emulsifier concentration up to $5 \mathrm{wt} . \%$ and then remained almost constant beyond this value. In this work, dependence of molecular weight on emulsifier concentration was not found explaining why $\eta$ is almost the same for different surfactant concentration (Figure 7).

3.2. Steady-Shear Flow Measurements. The flow viscosities for the polymer solutions in water at $25^{\circ} \mathrm{C}$ are depicted in Figures 8-12. Polymers characteristics correspond to Table 2. Figure 8 shows steady-shear viscosities for homopolymer PAM at various concentrations in water as a function of the shear rate. It is apparent that the viscosity increases as polymer concentration does. All curves exhibit a behavior predominantly Newtonian.

Figure 9 plots the viscosity against shear rate at different concentrations for multisticker PAM-co-DAM. As it can be seen, at low concentrations, shear viscosity does not exhibit significant changes with respect to the homopolymer behavior; however, after $4 \% \mathrm{wt}$, in the flow curves a Newtonian plateau is observed followed by a drop in viscosity. The behavior for the copolymer PAM-co-HDAM is similar; the viscosity curves are not shown but were used to obtain the zero-shear rate viscosity. Notice that for the same 


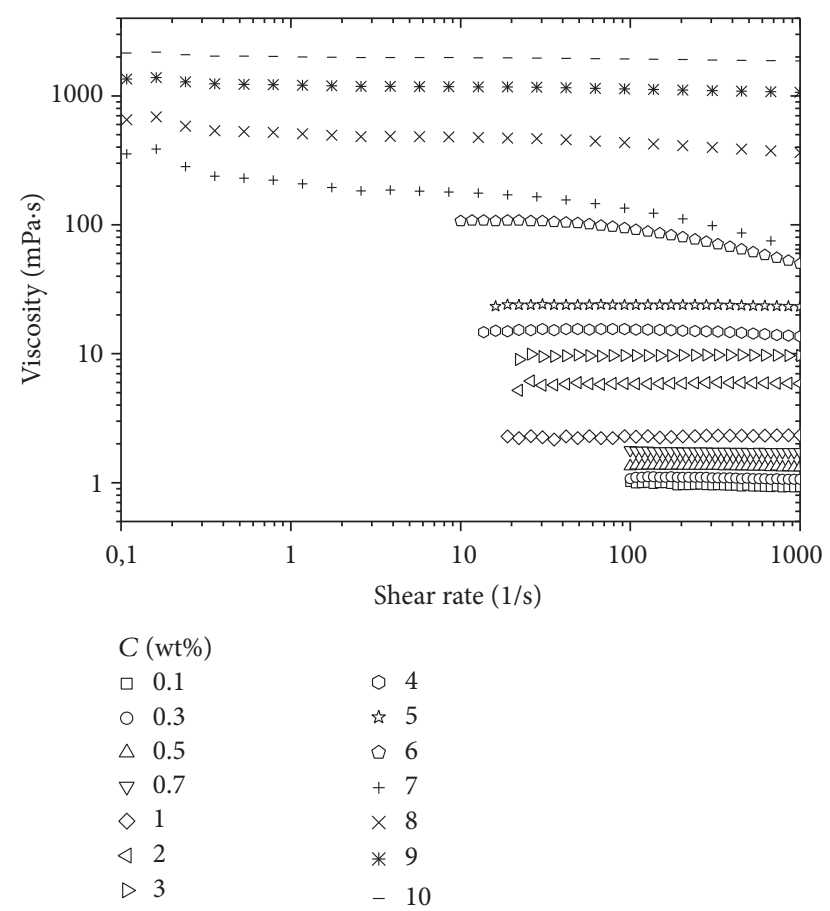

FigURE 8: Variation of the viscosity as a function the shear rate for various concentrations of PAM.

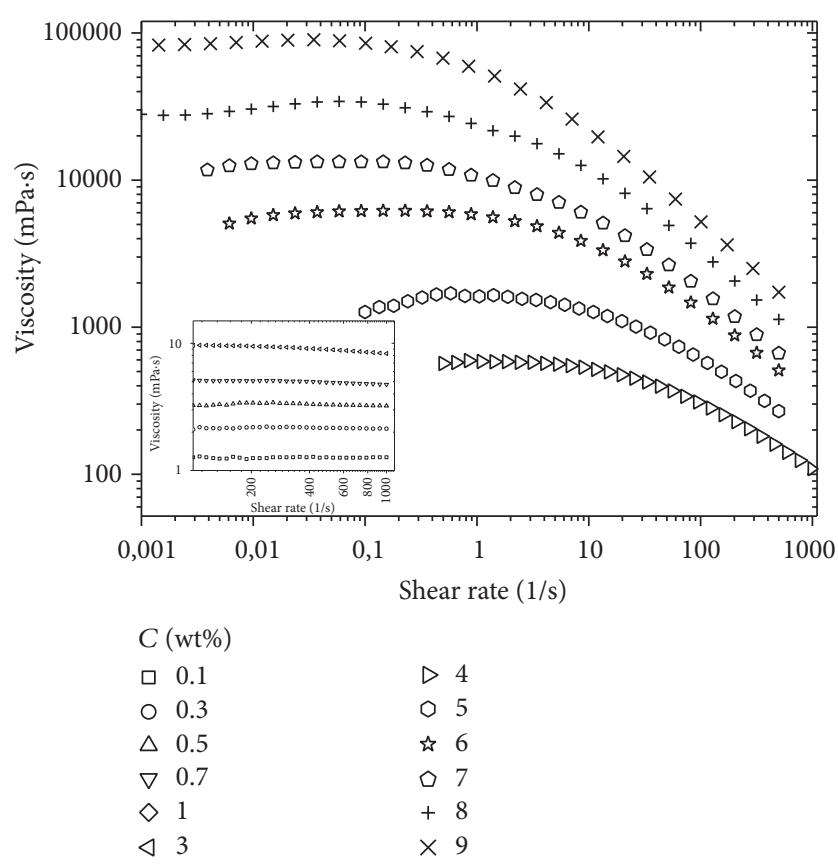

FIGURE 9: Variation of the viscosity as a function the shear rate for various concentrations of PAM-co-DAM.

solution concentration copolymers viscosity is significantly higher than that for homopolymer, confirming hydrophobic incorporation.

For the telechelic copolymer PAM/12 only at low concentrations a completely Newtonian behavior is observed (see Figure 10), and from $0.5 \%$ a slight shear-thinning is

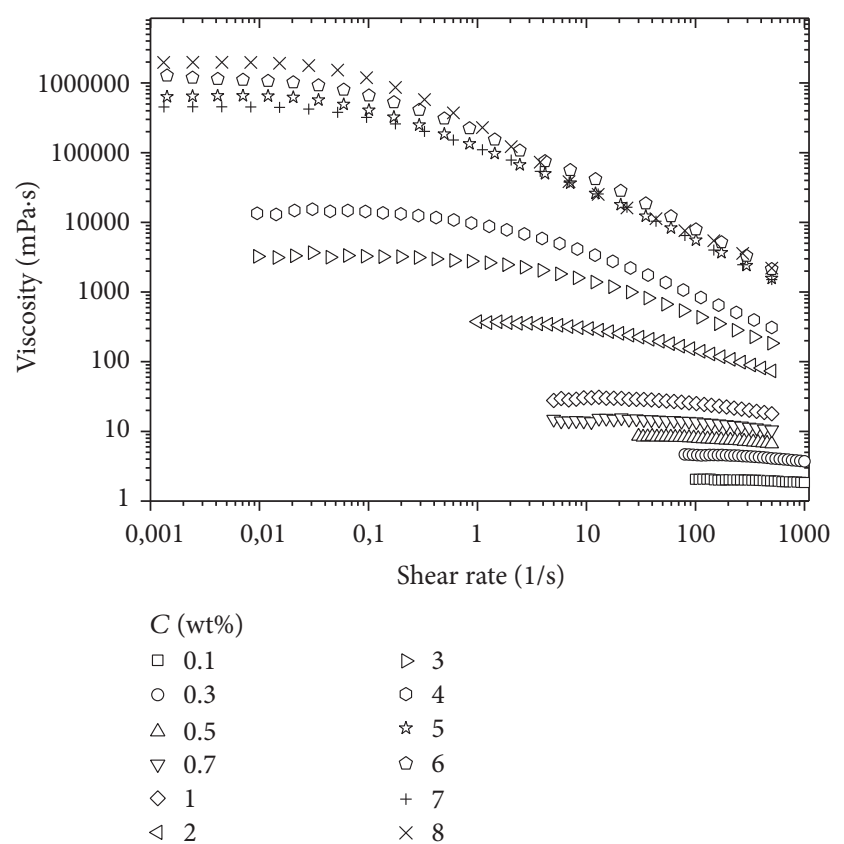

FIGURE 10: Variation of the viscosity as a function the shear rate for various concentrations of PAM/12.

observed. At high concentrations, it clearly shows a decrease of Newtonian plateau followed by a pronounced shearthinning; however, the traditional shear-thickening behavior is not observed. It is reported for telechelic polymers in aqueous solution that they have a shear-thickening effect because the end groups form micelles such as flowers; due to the increase in shear rate, they connect each other through the end groups of other polymer chains (bridges). This generates aggregates with a larger hydrodynamic volume affecting the solution viscosity. Finally, these aggregates break at higher shear rate and a shear-thinning is observed. In our case, shear-thickening effect is not observed, although the structure is of telechelic type. It is possible that the shear rate employed does not promote the formation of bridges between micellar flowers and, thus, the viscosity increase is not presented.

This behavior can be thought of as if hydrophobic groups were not incorporated. In that case, micellar flowers should not be formed; nevertheless, viscosity reached is really higher than that for homopolymer, indicating that the hydrophobe comonomer is incorporated and the telechelic structure is reached. Polymer behavior for PAM/16 is similar to PAM/12.

The mechanisms of association according to the viscosity behavior as a function of the shear rate can be observed in the nonlinear rheology. Typically, it is observed that the viscosity remains constant at a short interval at low shear rate, and at high shear rates the viscosity decreases. In our case, a Newtonian plateau is not observed, for the combined polymers (see Figure 11). A shear-thickening behavior, followed by an abrupt decrease in viscosity, is noticed. This behavior varies depending on the concentration, kind of associative polymer, and hydrophobic group. As mentioned, many authors agree that the shear-thickening effect is due to the shear stress 


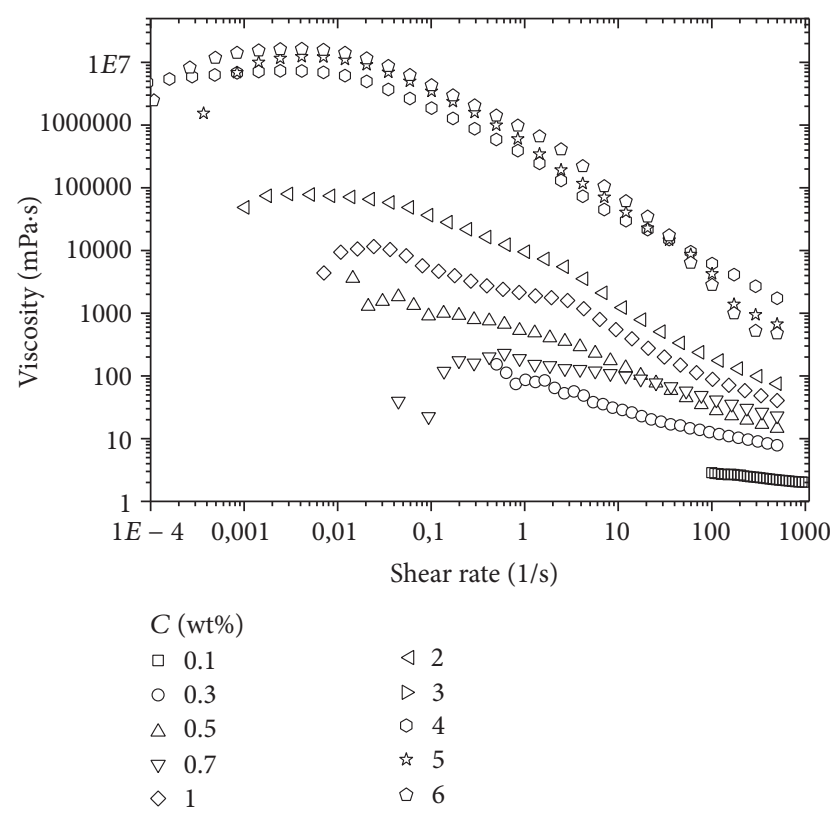

FIGURE 11: Variation of viscosity in terms of the shear rate for the aqueous solutions for combined copolymers PAM-co-DAM/12.

that promotes associations because more bridges are formed; this implies that the transient network formed has a greater hydrodynamic volume, so that the viscosity of the solution increases. In our case, bridges are not formed by micellar flower but a direct network for hydrophobic groups between and at the end of the chains. Finally, due to increased shear rate, these bridges break and the polymer chains are quickly oriented with the flow [45].

It is clear that the structure plays an important role. As it can be seen, shear viscosity increases according to the different structure obtained for the copolymers: multisticker, telechelic, and combined. It can be explained as follows: For telechelic copolymers, as it has been mentioned, the main chain bends easily for the hydrophobic groups located at the end can associate, then the loops formed increase viscosity. However, for multisticker copolymers, the hydrophobic groups are randomly distributed along the chain; therefore, it is difficult for the chain to bend and form a loop. Hydrophobic associations occur when the groups are far away from each other, and intermolecular interactions give a smaller size for the aggregates, as it has been demonstrated by molecular simulations [46]. For combined copolymers, due to the hydrophobic end groups, the formation of loops can occur and, also, the intramolecular hydrophobic groups can promote a net formation, explaining why a higher viscosity is observed (Figure 12).

The viscosity variation as function the polymer concentration was analyzed according to the hydrophobic group position; the viscosity was extrapolated to zero-shear rate except for combined polymers which was measured at low shear rate.

To analyze the results, it is necessary to indicate the main properties of rheological behavior for unmodified polymers, which present three regimes dependent on concentration [38, 47-49]:

(I) At very low concentrations, dilute regime, $\mathrm{C}<\mathrm{C \eta}$, where the rheological behavior is primarily controlled by intramolecular associations, is observed. Clusters of small size are formed, so that, the viscosity of the modified polymer is lower than unmodified polymer. $\mathrm{C \eta}$ is the critical concentration at which intermolecular associations form.

(II) A semidilute untangled regime is observed at $\mathrm{C} \eta<$ $C<C_{T}$. This regime is characterized by a rapid viscosity increase. This depends on the molecular weight of the polymer, the nature, concentration, and length of the hydrophobic group. $C_{T}$ is equivalent to $\mathrm{Ce}$ (for homopolymers), which represents the concentration at which the entanglements become elastically effective.

(III) A semidilute entangled regime, $C>C_{T}$, is observed. It can be safely assumed that, in the concentration range considered, the density of entanglements is much larger than that of hydrophobic associations.

Taking into consideration the applications, the three different architectures were studied, in order to compare the effects that structure has on viscosity. The Newtonian viscosity values are considered as the parameters to evaluate the thickening efficiency of the prepared associative polymers. Figures 13 and 14 show the variation of the zero-shear viscosity $\left(\eta_{0}\right)$ as a function of the polymer concentration $[C]$ for the telechelic, multisticker, and combined polymers, synthesized with different monomer length on the alkyl chain [C12 (Figure 10) and C16 (Figure 11)]. These copolymers are compared with the homopolymer. Three regimes can be identified for different homopolymer concentration. The aggregation concentration, $C^{*}$, is presented in $0.3 \%$ and the start concentration of crosslinking, $\mathrm{Ce}$, in $1.7 \%$. For associative polymers for series 12 , a short dilute regime is observed, because of their high molecular weight and hydrophobic contributions, so intramolecular associations are generated at lower concentrations. Semidiluted nonentangled regime is described by the model of reptation. $C_{T}$ for combined and telechelic polymers is presented at the same concentration (4\%), while $C_{T}$ of multisticker polymer is shifted at $6 \%$. This behavior is the result of combined and telechelic polymers generating a net with a hydrodynamic radius greater at lower concentrations than the multisticker polymer. Series 16 had a similar behavior. A diluted regime is observed; in this case, $C_{T}$ for combined and telechelic polymers is presented at $4 \%$, while the multisticker polymer $C_{T}$ is shifted to $5 \%$. A value near to four for the slope in the semidiluted entangled regimen curve is also observed which is representative for associative polymers. The slope curves of the semidilute regime varies depending on the hydrophobic groups and their localization [23-26].

Intramolecular hydrophobic associations occur at low polymer concentration and cause contraction of macromolecular spheres. In dilute regime, the viscosity of hydrophobically modified polymer is generally less than their 


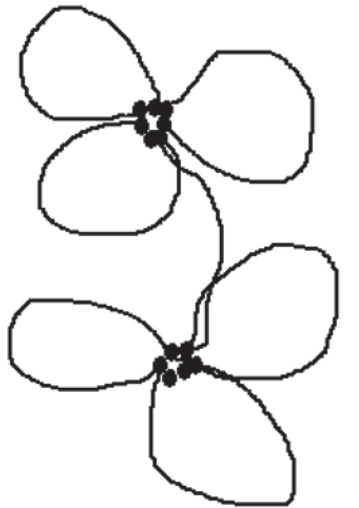

(a)

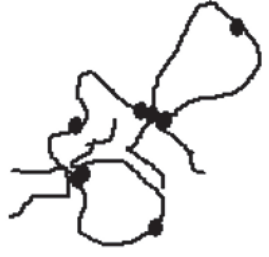

(b)

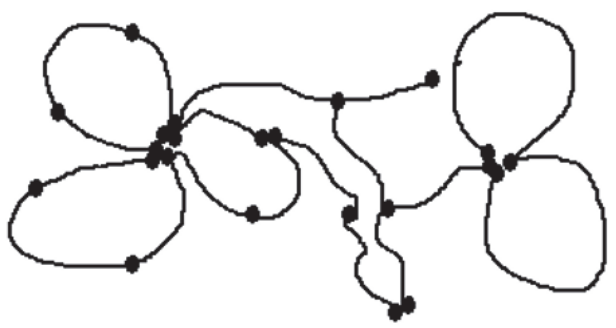

(c)

FIGURE 12: Schematic representation of the hydrophobic associations for the copolymers synthesized: (a) telechelic, (b) multisticker, and (c) combined.

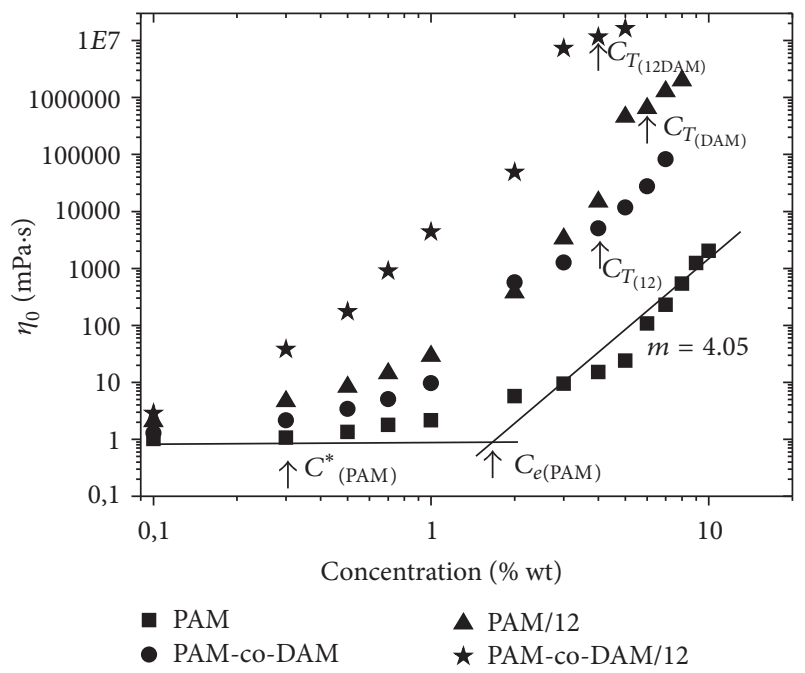

FIGURE 13: Variation of zero-shear viscosity $\left(\eta_{0}\right)$ as a function of polymer concentration $(C)$, for the synthesized polymers using the linear C12 hydrophobic group.

unmodified counterpart. When the polymer concentration increases and is greater than $C^{*}$, the viscosity of the modified polymer becomes higher than the unmodified polymer. In this stage, the intermolecular interactions become dominant with respect to intramolecular interactions; hence, a thickening effect is observed. In general, the concentration of the modified polymer $C^{*}$ is less than $C e$ of the modified polymer. The increase in viscosity from $C^{*}$ is more important when the polymer concentration is high. The thickening effect is moved towards smaller shear rates by the difference in viscosity between several orders of associative polymer and the unmodified counterpart. When intermolecular hydrophobic interactions are strong enough, they can reach the gel formation. Determining the value for $C^{*}$ is of paramount importance for the description of the rheological properties of associative polymers.

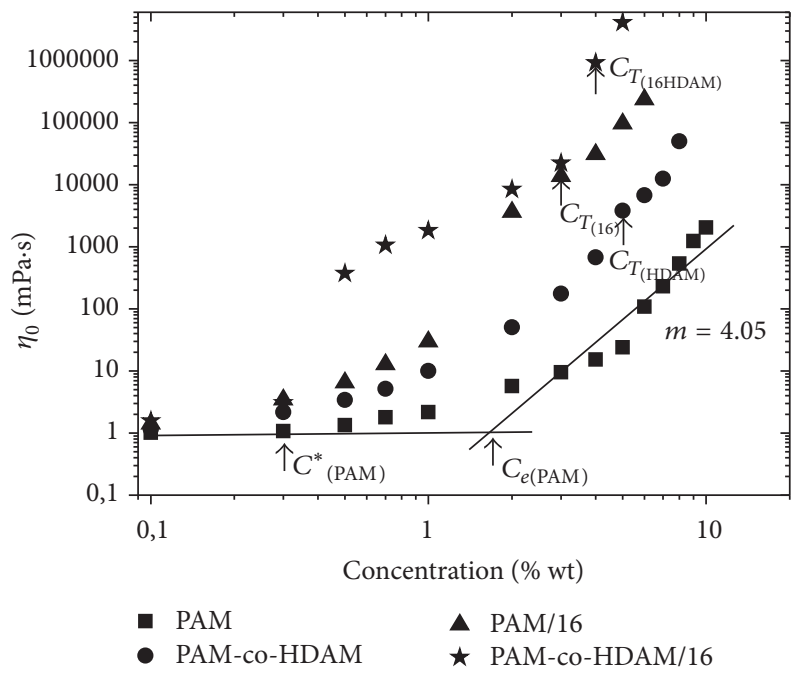

FIGURE 14: Variation of zero-shear viscosity $\left(\eta_{0}\right)$ as a function of polymer concentration $(C)$, for the synthesized polymers using the linear $\mathrm{C} 16$ hydrophobic group.

The following mentioned values of $\eta_{0}$ from aqueous solutions at $1 \%$ approximately of hydrophobically modified polyacrylamides which were synthesized by different methods of polymerization. Wever et al. [50] reported viscosities up to $1^{4} \mathrm{mPa} \cdot \mathrm{s}$ for polyacrylamides comb, line, and star, with a molecular weight of 230,000. Cram et al. [25] reported specific viscosities values up to 1 , for hydrophobically modified polyacrylamides synthesized by micellar polymerization, with a molecular weight of 96,000. Penott-Chang et al. [27] reported $\eta_{0}$ up to $1 \times 10^{3} \mathrm{mPa} \cdot \mathrm{s}$ for hydrophobically modified polyacrylamides synthesized by micellar polymerization, with a molecular weight of 4,200,000. Lu et al. [24] reported $\eta_{0}$ up to $1 \times 10^{6} \mathrm{mPa}$. for hydrophobically modified polyacrylamides synthesized by micellar polymerization, with a molecular weight of 340,000. Zhu et al. [23] reported $\eta_{0}$ up $800 \mathrm{mPa} \cdot \mathrm{s}$, for hydrophobically modified polyacrylamides 
synthesized by micellar polymerization, with a molecular weight of 2,300,000.

It is clearly known that viscosity of these polymers depends on several factors including molecular weight, hydrophobic content, type and location, method of polymerization, and polymer concentration; however, the results presented show that inverse emulsion polymerization is a suitable technique to synthesize these kinds of polymers.

\section{Conclusions}

Hydrophobic copolymers derived from acrylamide were satisfactorily synthesized by inverse emulsion polymerization. The hydrophobe content was limited to $1 \mathrm{~mol} \%$ and the incorporation level, determined by ${ }^{1} \mathrm{H}-\mathrm{NMR}$ spectroscopy, was close to the alimentation monomer feed. In order to analyze the influence of the length of the hydrophobe group in the rheological properties, homopolyacrylamide was also synthesized as reference.

The results showed that molecular weight of the final polymer increased by increasing emulsifier concentration and then remained almost constant without further increase. The data show that the molecular weight of final polymer is increased as monomer concentration increases. This aspect influences the behavior of viscosity of aqueous solution, because shear viscosity increases as the length of AM blocks is increased.

The viscosity of aqueous solutions is function of the polymeric structure; that is, polymers having higher viscosity are combined, followed by the telechelic and finally combined polymers. Multisticker solutions and telechelic polymers exhibit a Newtonian plate in viscosity as function of the shear rate, followed by a decrease in viscosity. For telechelic polymers, neither the plate nor the shear-thickening behavior is observed. Only two regimes dependent on concentration were determined, according to intra- and intermolecular associations of the different synthesized structures. Rheological behavior could not be observed in dilute regime, due to high viscosities of polymers solutions even at low concentrations.

\section{Conflicts of Interest}

The authors declare that they have no conflicts of interest.

\section{Acknowledgments}

The authors thank the financial support from PRODEP under Grant no. PROMEP/103.5/12/2116 and Judith CabelloRomero for NMR determinations. S. Carro thanks Erick Luna and Victor Amador for experimental assistance.

\section{References}

[1] E. J. Jiménez-Regalado, G. Cadenas-Pliego, M. Pérez-Álvarez, and Y. Hernández-Valdez, "Study of three different families of water-soluble copolymers: synthesis, characterization and viscoelastic behavior of semidilute solutions of polymers prepared by solution polymerization," Polymer, vol. 45, no. 6, pp. $1993-$ 2000, 2004.

[2] V. J. González-Coronel and E. J. Jiménez-Regalado, "Synthesis, characterization and rheological properties of three different microstructures of water-soluble polymers prepared by solution polymerization," Polymer Bulletin, vol. 62, no. 6, pp. 727-736, 2009.

[3] F. Candau and J. Selb, "Hydrophobically-modified polyacrylamides prepared by micellar polymerization," Advances in Colloid and Interface Science, vol. 79, no. 2-3, pp. 149-172, 1999.

[4] A. Hill, F. Candau, and J. Selb, "Properties of hydrophobically associating polyacrylamides: influence of the method of synthesis," Macromolecules, vol. 26, no. 17, pp. 4521-4532, 1993.

[5] W. D. Emmons and T. E. Stevens, "Acrylamide copolymer thickener for aqueous systems," U.S. Patent 4395 524, 1982.

[6] S. A. Ezzell and C. L. McCormick, "Water-soluble copolymers. 39. Synthesis and solution properties of associative acrylamido copolymers with pyrenesulfonamide fluorescence labels," Macromolecules, vol. 25, no. 7, pp. 1881-1886, 1992.

[7] J. J. Effing, I. J. McLennan, and J. C. T. Kwak, "Associative phase separation observed in a hydrophobically modified poly(acrylamide)/sodium dodecyl sulfate system," Journal of Physical Chemistry, vol. 98, no. 10, pp. 2499-2502, 1994.

[8] J. Bock, D. B. Siano, D. N. Schulz, S. R. Turner, and S.-L. Valint Jr., "Hydrophobically associating polymers," Polymeric Materials: Science and Engineering, vol. 55, article 355, 1986.

[9] J. Bock, P. L. Valint Jr., S. J. Pace, D. B. Siano, D. N. Schulz, and S. R. Turner, "Hydrophobically associating polymers," in WaterSoluble Polymers for Petroleum Recovery, G. A. Stahl and D. N. Schulz, Eds., pp. 147-160, Plenum Press, 1988.

[10] S. R. Turner, D. B. Siano, and J. Bock, "AcrylamideAlkylacrylamide Copolymers," U.S. Patent 4520 182, 1985.

[11] S. R. Turner, D. B. Siano, and J. Bock, "Micellar Process for the Production of Acrylamide-Alkylacrylamide Copolymers," U.S. Patent 4528 348, 1985.

[12] S. Evani, "Water Dispersible Hydrophobic Thickening Agent," U.S. Patent 4432 881, 1984.

[13] D. Hunkeler, "Synthesis and characterization of high molecular weight water-soluble polymers," Polymer International, vol. 27, no. 1, pp. 23-33, 1992.

[14] F. Stanley Jr., "Water-In-Oil Emulsions of Hydrophobe Association Polymers," U.S. Patent 4524 175, 1985.

[15] M. Pabon, J.-M. Corpart, J. Selb, and F. Candau, "Synthesis in inverse emulsion and associating behavior of hydrophobically modified polyacrylamides," Journal of Applied Polymer Science, vol. 91, no. 2, pp. 916-924, 2004.

[16] S. R. Turner, D. B. Siano, and J. Bock, "Microemulsion Process for Producing Acrylamide-Alkyl Acrylamide Copolymers," U.S. Patent 4521 580, 1985.

[17] J. W. Vanderhoff, E. B. Bradford, H. L. Tarkowski, J. B. Shaffer, and R. M. Wiley, "Inverse emulsion polymerization," in Polymerization and Polycondensation Processes, vol. 34 of Advances in Chemistry, pp. 32-51, American Chemical Society, Washington, DC, USA, 1962.

[18] J. W. Vanderhoff, D. L. Visioli, and M. S. El-Aasser, "Inverse emulsion polymerization of acrylamide: anomalous behavior of tetronic 1102 emulsifier," in Polymeric Materials Science and Engineering, vol. 54, pp. 375-380, 1986.

[19] C. Graillat, C. Pichot, A. Guyot, and M. S. El Aasser, "Inverse emulsion polymerization of acrylamide. I. Contribution to the 
study of some mechanistic aspects," Journal of Polymer Science Part A: Polymer Chemistry, vol. 24, no. 3, pp. 427-449, 1986.

[20] J. Hernández-Barajas and D. J. Hunkeler, "Inverse-emulsion polymerization of acrylamide using block copolymeric surfactants: mechanism, kinetics and modelling," Polymer, vol. 38, no. 2, pp. 437-447, 1997.

[21] E. Kobitstaya, Synthesis of hydrophobically modified polyacrylamide in inverse miniemulsion [Thesis], Universität Ulm, Moscu, Russia, 2008.

[22] P. L. Valint Jr., J. Bock, and D. N. Schulz, "Synthesis and characterization of hydrophobically associating polymers," in Polymeric Materials: Science and Engineering, vol. 57, p. 482, 1987.

[23] Z. Zhu, O. Jian, S. Paillet, J. Desbrières, and B. Grassl, "Hydrophobically modified associating polyacrylamide (HAPAM) synthesized by micellar copolymerization at high monomer concentration," European Polymer Journal, vol. 43, no. 3, pp. 824-834, 2007.

[24] C. Lu, W. Li, Y. Tan et al., "Synthesis and aqueous solution properties of hydrophobically modified polyacrylamide," Journal of Applied Polymer Science, vol. 131, no. 18, pp. 40754-40761, 2014.

[25] S. L. Cram, H. R. Brown, G. M. Spinks, D. Hourdet, and C. Creton, "Hydrophobically modified dimethylacrylamide synthesis and rheological behavior," Macromolecules, vol. 38, no. 7, pp. 2981-2989, 2005.

[26] S. Hietala, P. Mononen, S. Strandman et al., "Synthesis and rheological properties of an associative star polymer in aqueous solutions," Polymer, vol. 48, no. 14, pp. 4087-4096, 2007.

[27] E. K. Penott-Chang, L. Gouveia, I. J. Fernández, A. J. Müller, A. Díaz-Barrios, and A. E. Sáez, "Rheology of aqueous solutions of hydrophobically modified polyacrylamides and surfactants," Colloids and Surfaces A: Physicochemical and Engineering Aspects, vol. 295, no. 1-3, pp. 99-106, 2007.

[28] F. Bezzaoucha, P. Lochon, A. Jonquières, A. Fischer, A. Brembilla, and D. Ainad-Tabet, "New amphiphilic polyacrylamides: synthesis and characterisation of pseudo-micellar organisation in aqueous media," European Polymer Journal, vol. 43, no. 10, pp. 4440-4452, 2007.

[29] Q. Ye, X. Ge, H. Liu, H. Jia, W. He, and Z. Zhang, "Formation of monodisperse polyacrylamide particles by dispersion polymerization. I. Synthesis and polymerization kinetics," Journal of Macromolecular Science-Pure and Applied Chemistry, vol. 39, no. 6, pp. 545-556, 2002.

[30] Y. Li and J. C. T. Kwak, "Rheology and binding studies in aqueous systems of hydrophobically modified acrylamide and acrylic acid copolymers and surfactants," Colloids and Surfaces A: Physicochemical and Engineering Aspects, vol. 225, no. 1-3, pp. 169-180, 2003.

[31] J. Klein, G. Hannemann, and W.-M. Kulicke, "Zum Fließverhalten niedermolekularer Polyacrylamide in einem weiten Konzentrationsbereich," Colloid and Polymer Science Kolloid Zeitschrift \& Zeitschrift für Polymere, vol. 258, no. 6, pp. 719$732,1980$.

[32] I. Capek, "Inverse emulsion polymerization of acrylamide initiated by oil- and water-soluble initiators: effect of emulsifier concentration," Polymer Journal, vol. 36, no. 10, pp. 793-803, 2004.

[33] J. Herrera-Ordonez, O. Rivera, H. Maldonado-Textle, and J. C. Ramirez, "Kinetics of styrene emulsion polymerization above the critical micelle concentration: effect of the initial monomer concentration on the molecular weight," Journal of Polymer Science, Part A: Polymer Chemistry, vol. 43, no. 9, pp. 1963-1972, 2005.
[34] S. Carro, J. Herrera-Ordonez, and J. Castillo-Tejas, "On the evolution of the rate of polymerization, number and size distribution of particles in styrene emulsion polymerization above CMC," Journal of Polymer Science, Part A: Polymer Chemistry, vol. 48, no. 14, pp. 3152-3160, 2010.

[35] V. J. González Coronel and E. J. Jiménez-Regalado, "Rheological properties of three different microstructures of water-soluble polymers prepared by solution polymerization," Polymer Bulletin, vol. 67, no. 2, pp. 251-262, 2011.

[36] J. Bock, D. B. Siano, P. L. Valint Jr., and S. J. Pace, "Structure and properties of hidrophobically associated polymers," in Polymeric Materials: Science and Engineering, vol. 57, pp. 487491, 1987.

[37] E. Volpert, J. Selb, and F. Candau, "Associating behaviour of polyacrylamides hydrophobically modified with dihexylacrylamide," Polymer, vol. 39, no. 5, pp. 1025-1033, 1998.

[38] E. Volpert, J. Selb, and F. Candau, "Influence of the hydrophobe structure on composition, microstructure, and rheology in associating polyacrylamides prepared by micellar copolymerization," Macromolecules, vol. 29, no. 5, pp. 1452-1463, 1996.

[39] W. Xue, I. W. Hamley, V. Castelletto, and P. D. Olmsted, "Synthesis and characterization of hydrophobically modified polyacrylamides and some observations on rheological properties," European Polymer Journal, vol. 40, no. 1, pp. 47-56, 2004.

[40] L. Ouyang, L. Wang, and F. J. Schork, "Synthesis and nucleation mechanism of inverse emulsion polymerization of acrylamide by RAFT polymerization: a comparative study," Polymer, vol. 52, no. 1, pp. 63-67, 2011.

[41] F. Candau, Y. S. Leong, and G. J. Pouyet, "Inverse microemulsion polymerization of acrylamide; Characterization of the waterin-oil microemulsions and the final microlatexes," Journal of Colloid and Interface Science, vol. 114, no. 2, pp. 167-183, 1984.

[42] F. Candau, Z. Zekhnini, and J. P. Durand, "Copolymerization of water-soluble monomers in nonionic bicontinuous microemulsions," Journal of Colloid and Interface Science, vol. 114, no. 2, pp. 398-408, 1986.

[43] I. Capek, L. Fialová, and D. Berek, "On the kinetics of inverse emulsion polymerization of acrylamide," Designed Monomers and Polymers, vol. 11, no. 2, pp. 123-137, 2008.

[44] M. Barari, M. Abdollahi, and M. Hemmati, "Synthesis and characterization of high molecular weight polyacrylamide nanoparticles by inverse-emulsion polymerization," Iranian Polymer Journal, vol. 20, no. 1, pp. 65-76, 2011.

[45] C. Chassenieux, T. Nicolai, and L. Benyahia, "Rheology of associative polymer solutions," Current Opinion in Colloid and Interface Science, vol. 16, no. 1, pp. 18-26, 2011.

[46] J. Castillo-Tejas, O. Castrejón-González, S. Carro, V. GonzálezCoronel, J. F. J. Alvarado, and O. Manero, "Associative polymers. Part III: shear rheology from molecular dynamics," Colloids and Surfaces A: Physicochemical and Engineering Aspects, vol. 491, pp. 37-49, 2016.

[47] M. Camail, A. Margaillan, I. Martin, A. L. Papailhou, and J. L. Vernet, "Synthesis of $\mathrm{N}$-alkyl- and $\mathrm{N}$-arylalkylacrylamides and micellar copolymerization with acrylamide," European Polymer Journal, vol. 36, no. 9, pp. 1853-1863, 2000.

[48] V. Castelletto, I. W. Hamley, W. Xue, C. Sommer, J. S. Pedersen, and P. D. Olmsted, "Rheological and structural characterization of hydrophobically modified polyacrylamide solutions in the semidilute regime," Macromolecules, vol. 37, no. 4, pp. 1492-1501, 2004 . 
[49] A. A. Abdala, W. Wu, K. R. Olesen, R. D. Jenkins, A. E. Tonelli, and S. A. Khan, "Solution rheology of hydrophobically modified associative polymers: effects of backbone composition and hydrophobe concentration," Journal of Rheology, vol. 48, no. 5, pp. 979-994, 2004.

[50] D. A. Z. Wever, F. Picchioni, and A. A. Broekhuis, "Branched polyacrylamides: synthesis and effect of molecular architecture on solution rheology," European Polymer Journal, vol. 49, no. 10, pp. 3289-3301, 2013. 

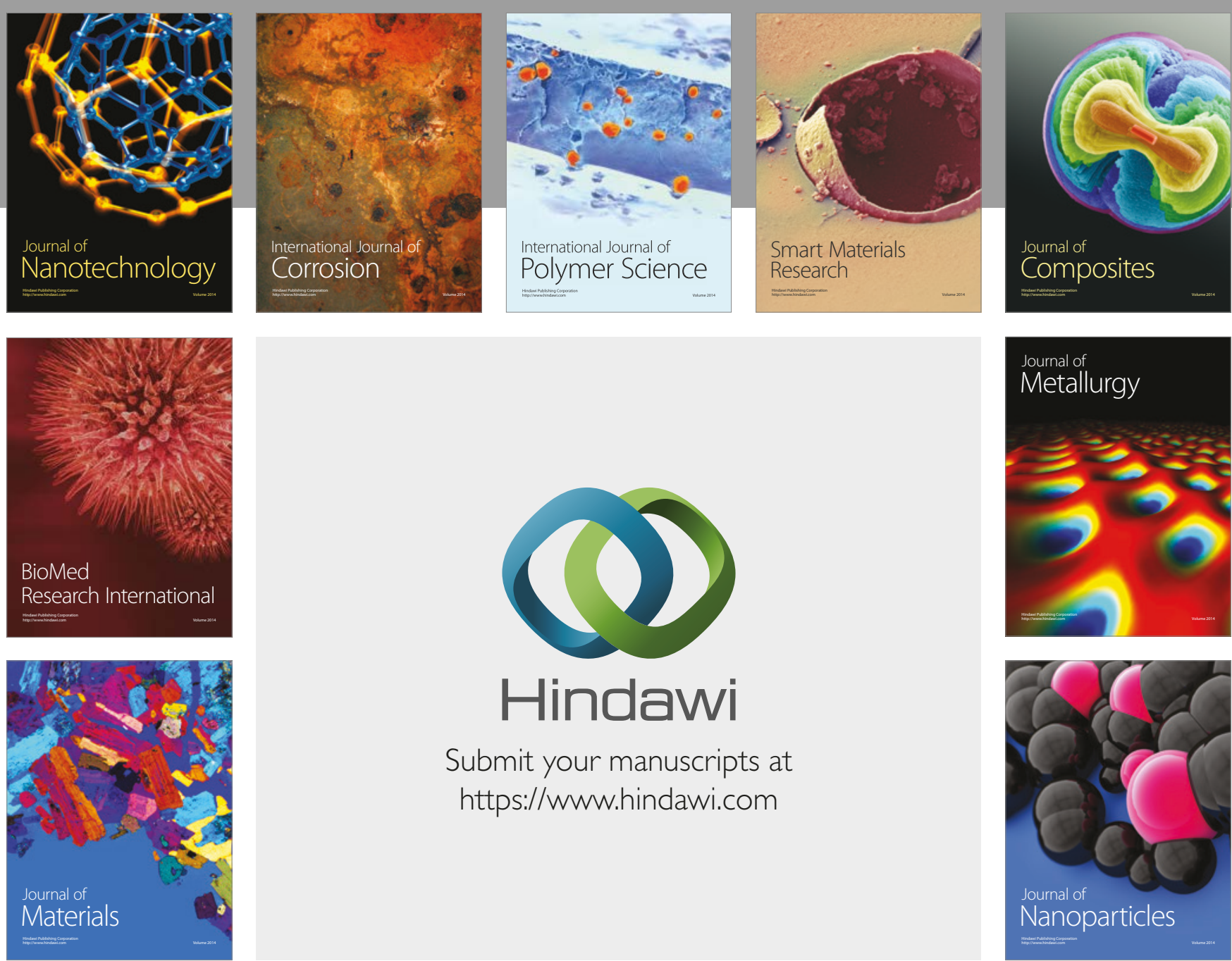

\section{Hindawi}

Submit your manuscripts at

https://www.hindawi.com
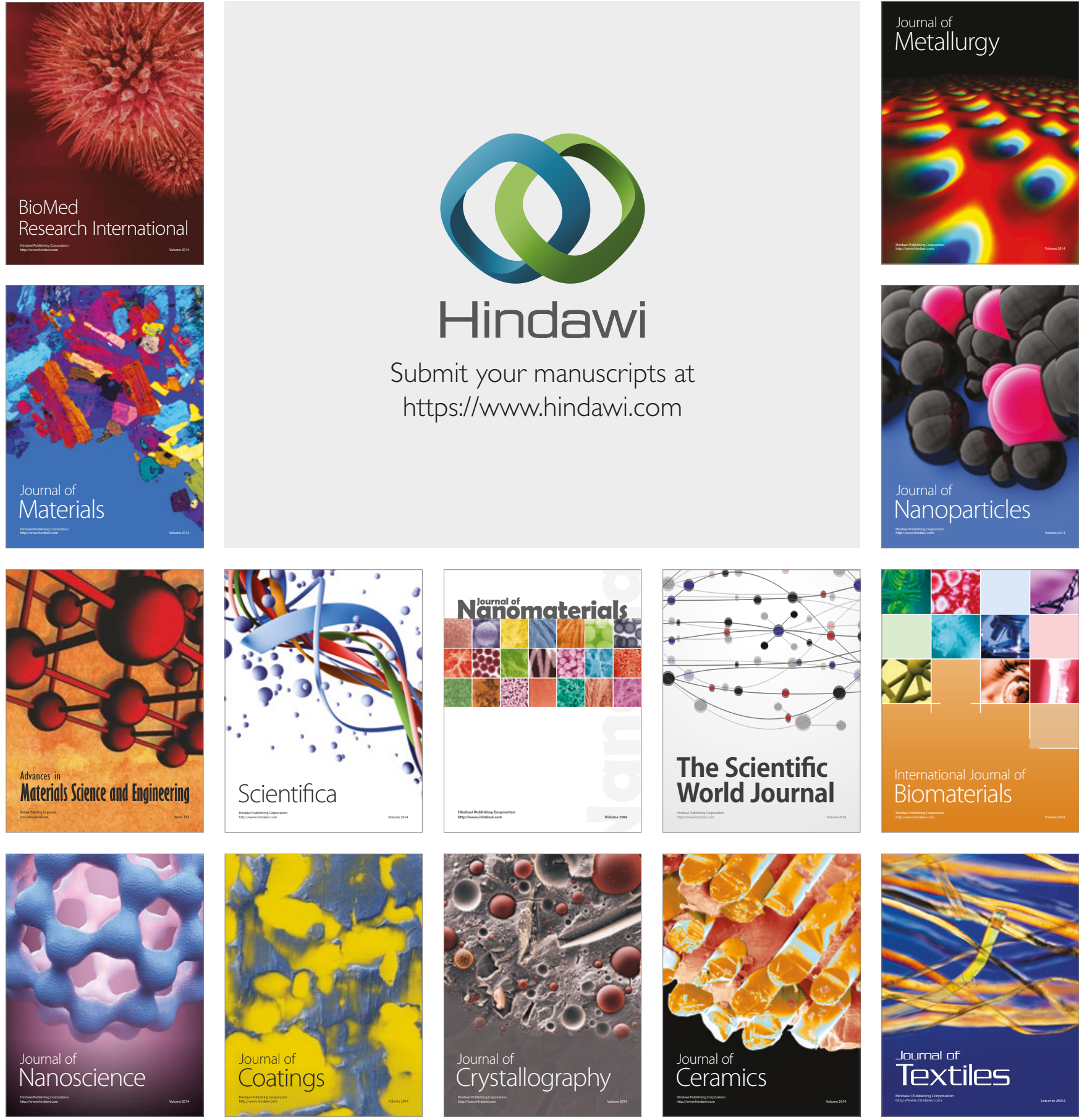

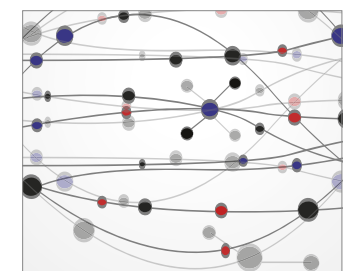

The Scientific World Journal
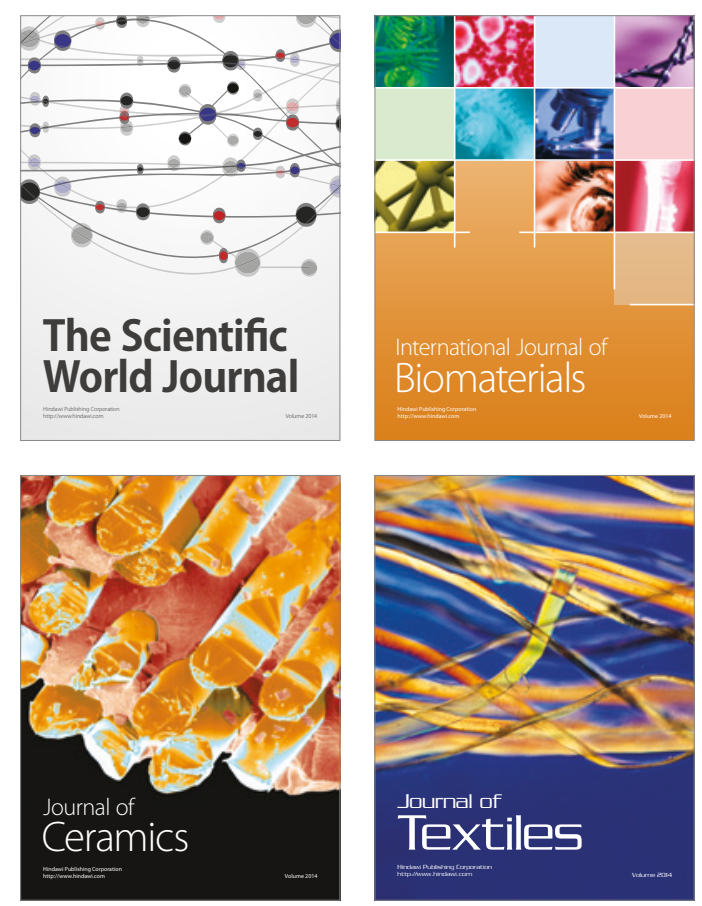